\title{
Reparación de perforación septal con técnica de colgajos pediculados asistida con endoscopio
}

\author{
Nasal septal perforation repair using pediculated graft technique assisted by \\ endoscope
}

\author{
Rodolfo Nazar $\mathbf{S}^{1}$, Gonzalo Ortega F², Alejandra Salinas G³ , Carla Parra A², Alfredo Naser G'.
}

\begin{abstract}
RESUMEN
La perforación septal corresponde a la comunicación entre ambas fosas nasales por un defecto del tabique nasal. Si bien la mayoría son asintomáticas, pueden presentarse como una rinitis costrosa, obstrucción nasal, epistaxis recidivante o sibilancias nasales. Su origen es predominantemente iatrogénico o idiopático, no obstante pueden presentar diversas etiologías. Es necesario determinar esto para resolver la necesidad de cirugía y definir la técnica quirúrgica más adecuada. En el siguiente trabajo presentamos 2 casos clínicos de perforación septal manejados con la técnica de avance de colgajos de mucosa de Fairbanks modificada, interponiendo además cartílago tragal.
\end{abstract}

Palabras clave: Nariz, tabique nasal, perforaciones septales, tratamiento quirúrgico, fairbanks.

\section{SUMMARY}

The septal perforation is a communication between both nostrils through a nasal septal defect. Most of these defects are asymptomatic, although some may present as crusty rhinitis, nasal obstruction, recurrent epistaxis or nasal wheeze. Its origin is predominantly iatrogenic or idiopathic; however it may have several etiologies. It's fundamental to determine the cause of the septal perforation, in order to address the need for surgery and define the most appropriate surgical technique. We report 2 cases of septal perforations which were resolved with modified Fairbanks technique interposing tragal cartilage.

Key words: Nose, nasal septum, septal perforations, surgical treatment.

\footnotetext{
Médico Otorrinolaringólogo, Servicio de Otorrinolaringología Hospital Clínico de la Universidad de Chile.

2 Interno de Medicina Universidad de Chile

3 Interno de Medicina Universidad de La Frontera. Servicio de Otorrinolaringología Hospital Clínico de la Universidad de Chile.
} 


\section{INTRODUCCIÓN}

El tabique nasal es una estructura que divide las 2 fosas nasales. Está compuesto por hueso y cartílago, contribuye al soporte de la nariz y a su conformación externa. Una perforación septal consiste en una comunicación de ambas fosas nasales a través del tabique nasal, por sus porciones cartilaginosas, óseas o ambas. El cartílago septal es la zona más frecuentemente comprometida ${ }^{1}$.

La perforación septal no es una patología en sí, sino una expresión de una amplia gama de condiciones que pueden afectar el tabique nasal'.

Las principales etiologías pueden ser traumáticas, iatrogénicas, idiopáticas, infecciosas, neoplásicas, por agentes químicos 0 enfermedades inflamatorias, siendo la iatrogénica poseptoplastía o rinoseptoplastía la causa más frecuente (Tabla $1)^{2-5}$. Dentro de las etiologías que han cobrado importancia en las últimas décadas destaca la necrosis isquémica por cocaína, presente en pacientes con un consumo prolongado y frecuente (mayor a tres semanas) $)^{6,7}$. Debido a esto, es de gran importancia realizar un estudio minucioso previo a un posible tratamiento quirúrgico, ya que una patología crónica subyacente 0 el consumo crónico de cocaína podría generar la recurrencia del cuadro.

Una comunicación directa de ambas fosas nasales compromete la funcionalidad de la nariz, ya que se generan cambios en la corriente de aire inspirado junto con una disminución de la velocidad global del flujo aéreo, pasando de un flujo laminar a uno turbulento ${ }^{3}$. Las manifestaciones clínicas son variadas, presentándose con mayor frecuencia obstrucción nasal, costras endonasales, epistaxis, rinorrea y sibilancias ${ }^{2,3,5}$.

Las perforaciones anteriores y de mayor diámetro son las más sintomáticas. Perforaciones pequeñas son aquellas con un diámetro menor de $0,5 \mathrm{~cm}$., las medianas entre 0,5 y $2 \mathrm{~cm}$. y las grandes mayores a $2 \mathrm{~cm}^{2}$.

El diagnóstico se realiza mediante una exhaustiva anamnesis y un acucioso examen físico, en donde es posible evidenciar algún factor desencadenante como traumas o cirugías previas y la perforación septal en sí, con la presencia de costras y epistaxis ${ }^{2,3}$.

En cuanto a los estudios complementarios destaca la tomografía computarizada de nariz y cavidades paranasales, la cual permite medir los diámetros de la perforación, su relación con el borde caudal del tabique nasal, así como constatar alguna desviación septal concomitante. Por otro lado la visualización con fibra óptica permite obtener una visión más detallada y completa de la anatomía, además de definir el estado de la mucosa adyacente, que podrá emplearse para reparar el tabique nasal ${ }^{2-4}$.

El tratamiento de una perforación septal está indicado en pacientes sintomáticos. Este puede ser médico 0 quirúrgico ${ }^{8,9}$.

Respecto al manejo médico, el uso de cremas humectantes, lavados con solución salina y ungüentos con antibióticos pueden ser capaces de reducir e incluso cesar la sintomatología 8,9 . Se

Tabla 1. Causas de perforación septal

\begin{tabular}{|lll|}
\hline Traumáticas / latrogénicas & Enfermedades inflamatorias & Infecciosas \\
\hline Rino/septoplastía & Sarcoidosis & Sífilis \\
Cauterizaciones septales & Enfermedad de Crohn & Tuberculosis \\
Bilaterales & Dermatomiositis & Fiebre tifoidea \\
Taponamiento con presión & Lupus Eritematoso & Micosis nasal \\
Elevada & Sistémico & Rinoescleroma \\
Hematoma o absceso septal & Artritis reumatoidea & Difteria \\
Cuerpo extraño & Enfermedad de Wegener & \\
& Vasculitis & \\
\hline Neoplásicas & Agentes químicos & Idiopática \\
\hline Carcinoma & Cocaína & Ulcera de Hajek \\
Leucemia & Vasoconstrictores locales & \\
Granulomas & Partículas de polvo, cal, & \\
& cemento, vidrio, sal, jabón & \\
& Pilas eléctricas, baterías & \\
\hline
\end{tabular}


puede intentar cerrar el defecto con prótesis siliconadas (o botones septales), sin embargo éstas requieren aseo constante e incluso reinstalaciones repetitivas, las cuales pueden ser mal toleradas por el paciente e incluso generar obstrucción nasal 0 aumentar el tamaño del defecto ${ }^{8}$. Este método es utilizado generalmente cuando existe una contraindicación quirúrgica.

El manejo quirúrgico ofrece los mejores resultados fisiológicos. Se indica en todas las perforaciones septales sintomáticas, estando contraindicada en casos de infección, neoplasia, enfermedad granulomatosa, consumo de cocaína 0 patología vascular ${ }^{2,5,9}$. Existen numerosas técnicas quirúrgicas para reparar la perforación septal. Estas consisten principalmente en realizar un avance o rotación de un colgajo de mucosa bien vascularizado proveniente de septum restante, piso nasal, pared lateral de la fosa nasal, cornete inferior 0 mucosa labial, con interposición de un injerto de sostén autólogo $0^{2-5,10-12}$.

La técnica quirúrgica puede ser cerrada 0 abierta. La técnica abierta fue descrita por primera vez por Strelzow Goodman en 1978 y 1982 en pacientes con perforaciones mayores, ofreciendo tasas de más de $90 \%$ de éxito en defectos menores de $3 \mathrm{~cm}^{13-15}$. En relación a la cerrada, se describe desde 1947 y ha ido progresando hasta la actualidad, reservándose para perforaciones de tamaño pequeño $0^{2-5,10-12}$.

En el siguiente trabajo expondremos 2 casos de perforación septal en los cuales se utiliza la técnica cerrada de cierre de perforaciones del tabique nasal mediante colgajos en puente descrita por primera vez por Fairbanks (Figuras 1 y 2, esquema modificado tomado de Neumann y cols $^{16}$ ). A esta técnica se le realizaron algunas modificaciones. La técnica original descrita por Fairbanks, consiste

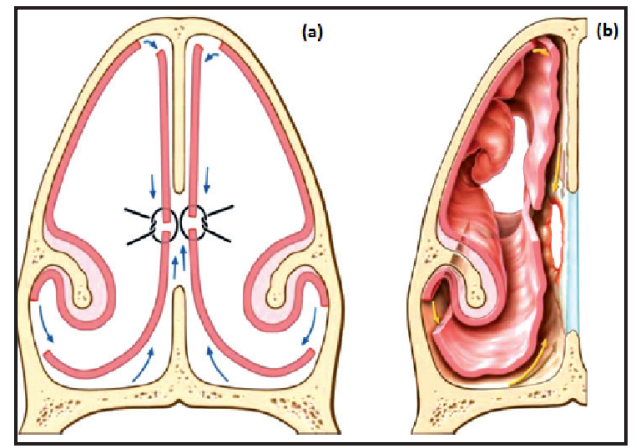

Figura 1. Principio de la técnica de colgajo en puente. Cortes transversal (a) y esquema 3D (b) ${ }^{16}$.
Figura 2. (a) Movilización de mucoperiostio ventral y craneal al defecto a través de incisión transfixiante, (b) movilización de mucoperiostio del suelo de la fosa nasal, (c) movilización de mucoperiostio partiendo del suelo de la fosa nasal caudalmente al defecto, aquí se realizó una incisión vertical posterior al defecto, (d) movilización de mucoperiostio partiendo del techo de la fosa nasal craneal al defecto, aquí se realizó una incisión horizontal superior al defecto y otra vertical posterior a este , (e) puntos colchoneros en el cierre de la mucosa de la perforación. Esquema modificado tomado de Neumann y cols ${ }^{16}$.

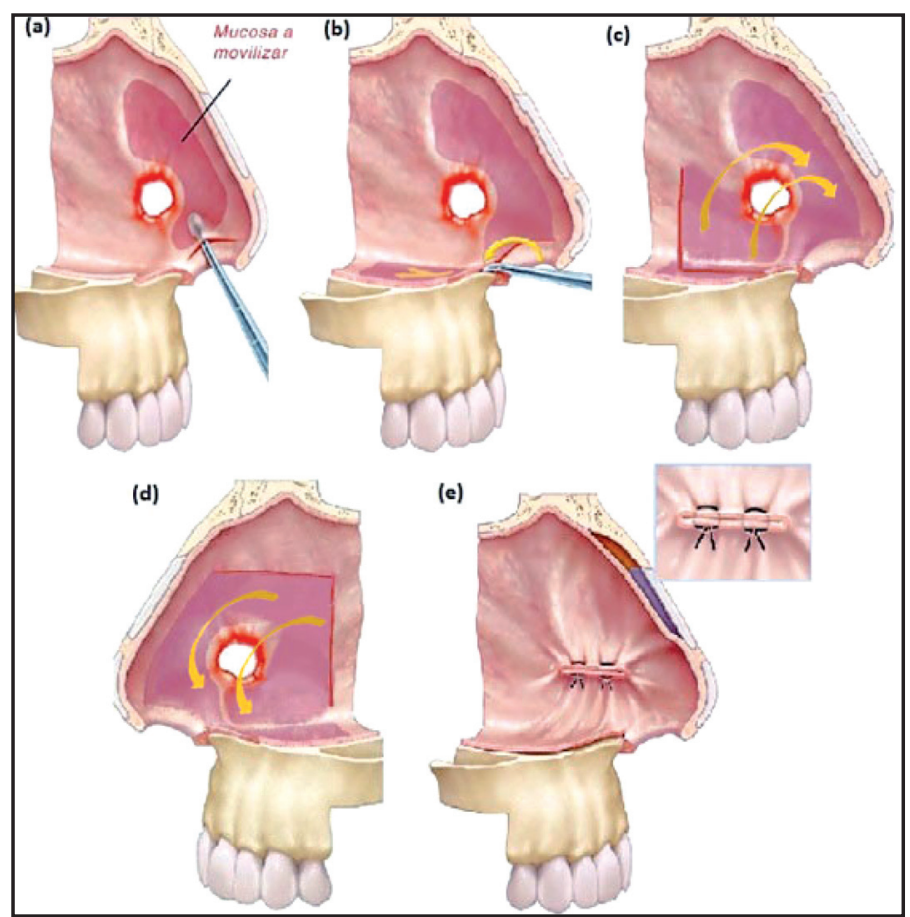


en hacer 2 incisiones en la mucosa de cada fosa nasal: una superior y otra caudal a la perforación, y de esta manera avanzar la mucosa para cerrar el defecto.

En los 2 casos presentados sólo se realizó una incisión horizontal inferior (en fosa nasal derecha) o superior (en fosa nasal izquierda) al defecto. Esta incisión horizontal se unió en ambos lados con otra vertical posterior a la perforación septal, quedando finalmente una incisión en forma $\mathrm{L}$.

Esto permitió una adecuada rotación de los colgajos. Además se conservó la mucosa del margen anterior de la perforación, evitando nueva patología del marco caudal anterior.

\section{CASO $\mathbf{N}^{0} 1$}

Paciente de sexo masculino de 32 años consulta en el Servicio de Otorrinolaringología del Hospital Clínico de la Universidad de Chile por obstrucción nasal de larga data, sin antecedentes de alergias ni de consumo de drogas. Refiere haber sufrido una fractura nasal hace 15 años, la cual se manejó en forma conservadora. El paciente evoluciona con episodios recurrentes de epistaxis, obstrucción nasal, rinorrea y formación de costras. Al examen físico se evidencia una perforación septal anterior de $1 \times 1 \mathrm{~cm}$. con costras a su alrededor. Se realiza tomografía computarizada de nariz y cavidades paranasales que evidencia una perforación septal anterior (Figura 3).

Se evalúa al paciente por reumatología, quienes descartan enfermedades sistémicas como causa de la perforación. Se biopsia la mucosa nasal descartando patologías granulomatosa y vasculítica. Se diagnostica una perforación septal sintomática de causa posiblemente traumática por lo que se indica su resolución quirúrgica con la técnica de Fairbanks modificada (Figura 4); se toma injerto tragal derecho para interponerlo en el defecto y así ayudar al cierre de la perforación. El procedimiento resulta exitoso y sin incidentes.

El paciente evoluciona favorablemente, se retira Meroce ${ }^{\circledR}$ y las placas de silicona durante las 2 primeras semanas del posoperatorio. La mucosa septal se observa íntegra y sin solución de continuidad. Paciente con control 1 año de posoperado con perforación cerrada.

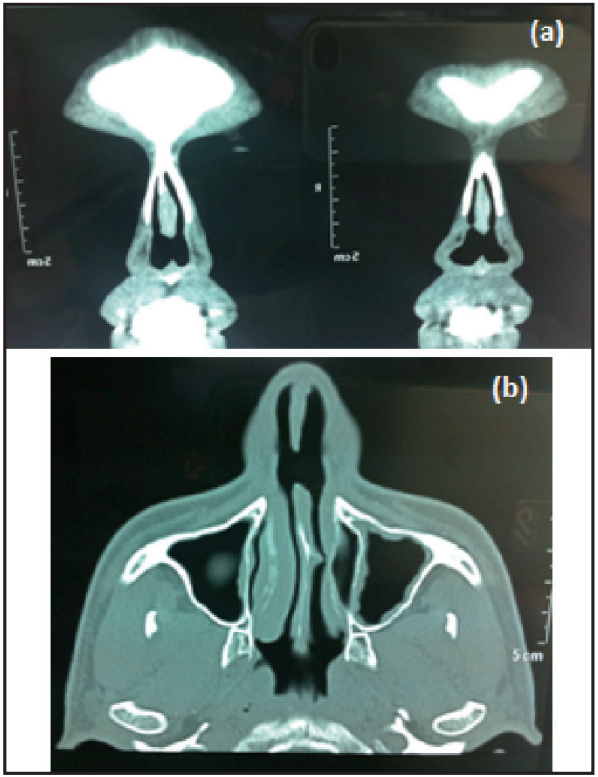

Figura 3: Tomografía computarizada de cavidades paranasales, cortes coronal (a) transversal (b). Se observa una solución de continuidad del septum cartilaginoso en su porción anterior de $1 \times 1 \mathrm{~cm}$. Se observa además un espolón septal a izquierda.

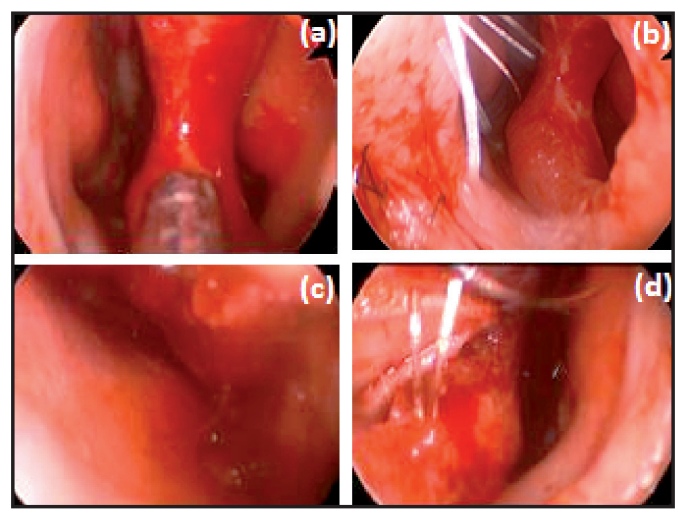

Figura 4: Imágenes tomadas mediante cirugía endoscópica. (a) Perforación septal, se observan ambas fosas nasales a través de la perforación, (b) visión endoscópica de perforación septal vista por fosa nasal derecha, (c) fosa nasal derecha con defecto septal reparado (septum a derecha), (d) fosa nasal izquierda con defecto septal reparado (septum a izquierda).

\section{CASO $N^{0} 2$}

Paciente de sexo masculino de 42 años de edad, sin antecedentes médicos relevantes, consulta al Servicio de Otorrinolaringología del Hospital Clínico de la Universidad de Chile por obstrucción nasal 


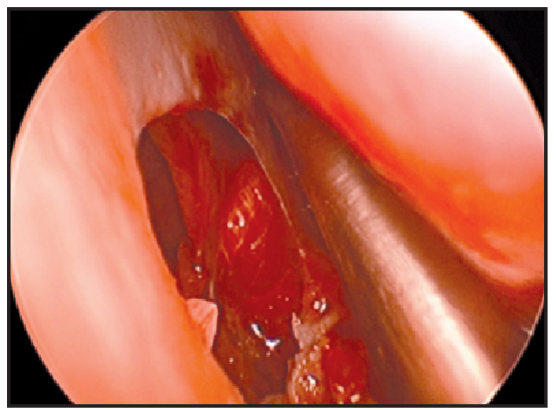

Figura 5. Visión endoscópica de perforación septal, vista por fosa nasal izquierda, una vez despegada la mucosa durante la cirugía de reparación.

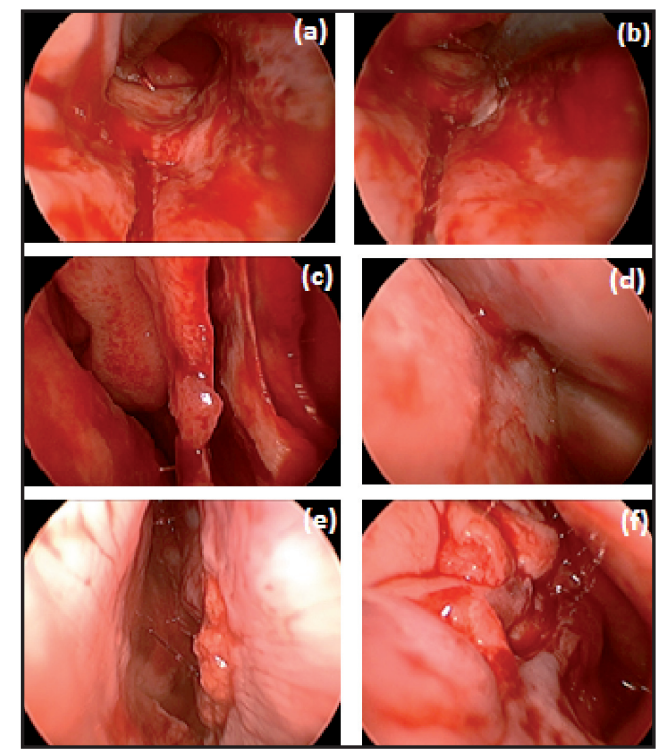

Figura 6. Imágenes tomadas mediante cirugía endoscópica. (a) y (b)utilización de la aguja de Tungsteno, para tallar las incisiones en forma de $L$ del colgajo a nivel del piso y tabique de la fosa nasal derecha, (c) visualización de la perforación septal y de la mucosa tabique nasal despegada a ambos lados, (d) confección de los túneles de Cottle mediante aspirador decolador (tabique a izquierda, mucosa a derecha), (e) fosa nasal derecha con defecto septal reparado (tabique nasal a derecha, con material de sutura), (f) fosa nasal izquierda con defecto septal reparado( tabique nasal a izquierda).

bilateral asociada a rinorrea mucosa de 1 año de evolución. No presenta alergias ni consumo de medicamentos o drogas. Refiere haber sufrido un accidente de tránsito 20 años atrás. Al examen físico se evidencia una perforación septal anterior de $1 \mathrm{x}$ $2 \mathrm{~cm}$ asociada a septodesviación a izquierda con signos de fractura nasal antigua (Figura 5). Se realiza tomografía computarizada de nariz y cavidades paranasales que evidencia una perforación septal anterior de $1 \times 2 \mathrm{~cm}$.

Se toma biopsia de la mucosa de la perforación, descartando patología reumatológica. Se diagnostica una perforación septal sintomática de causa posiblemente traumática. Se indica resolución quirúrgica con la técnica de Fairbanks modificada (Figura 6); se toma injerto tragal para interponerlo en el defecto. El procedimiento resulta exitoso y sin incidentes.

El paciente evoluciona favorablemente, se retira Merocel ${ }^{\circledR}$ y las placas de silicona durante las 2 primeras semanas del posoperatorio. La mucosa septal se observa íntegra y sin solución de continuidad. Paciente con control 6 meses de posoperado con perforación cerrada.

\section{DISCUSIÓN}

Existen más de 40 técnicas quirúrgicas para reparar defectos del tabique nasal. Esto evidencia que actualmente no hay disponible una técnica que sea capaz de cerrar todo tipo de perforación septal. Además, como una de las principales etiologías es la iatrogenia poscirugía septal, al momento de plantear una técnica quirúrgica para reparar el defecto septal, la fosa nasal posee menor tejido y un aporte vascular limitado. Por otro lado, una parte considerable de las perforaciones septales no son reparadas ya que las opciones quirúrgicas disponibles son técnicamente difíciles de realizar, requiriendo de entrenamiento y experiencia ${ }^{16,17}$.

Existen 2 recomendaciones realizadas por Fairbanks que apuntan a prevenir la perforación septal como complicación posoperatoria de una septoplastía. Una es que el aporte sanguíneo del cartílago septal depende de la mucosa subyacente, y éste se verá alterado si se daña la mucosa en áreas bilaterales del septum. Otro postulado es que si llega a generarse una perforación septal en una resección submucosa, aunque sea pequeña, debe repararse lo antes posible, ya que el proceso de sanación normal provocará que aumente el defecto más que cerrarlo $0^{5,9,18}$.

La técnica utilizada en nuestros casos para reparar la perforación septal fue la cerrada de 
Fairbanks, por tratarse de perforaciones anteriores, menores de $2 \mathrm{~cm}$. En esta técnica se unen los principios de un colgajo en puente junto con movilización endonasal bilateral de la mucosa, con lo cual se debe tener en consideración la vascularización del colgajo por avance $5^{5,9,16,18}$. Se asocia un injerto de tejido conjuntivo interpuesto mayor al defecto, como por ejemplo cartílago autólogo o fascia, a los colgajos de mucosa intranasal, generando una barrera entre las dos aletas reparadas $5,9,16,18$. Como la movilización de los colgajos es hacia craneal y caudal, un elemento clave de la técnica es evaluar las dimensiones del defecto septal en su altura vertical y la altura del tabique, ya que si la altura vertical del defecto es mayor a la mitad de la altura del tabique, el éxito de la cirugía disminuye $e^{5,9,16,18}$. No debe quedar expuesto cartílago septal al mismo nivel ya que podría formarse una nueva perforación debido a la falta de irrigación ${ }^{18}$. Se debe tener cuidado de no unir ambos colgajos entre sí al momento de realizar las suturas ${ }^{5,9}$.

Otra ventaja de esta cirugía es la capacidad de utilizar la endoscopía endonasal, ya que otorga al cirujano una visión clara y magnificada de la fosa nasal. Los endoscopios permiten que toda la operación se realice a través de la fosa nasal, sin necesidad de hacer incisiones en la piel que podrían dañar la irrigación columenar, comprometiendo la vitalidad de los colgajos. Su utilidad consiste en realizar resecciones precisas de forma mínimamente invasiva, conservando en lo posible las estructuras anatómicas sanas y evitando complicaciones $^{19}$. Por otro lado, la utilización de fibra óptica, permite una mayor precisión en la confección de colgajos, así como un trabajo en profundidad de la fosa nasal, mucho más preciso (Figura 6 (a) y (b)).

En relación a los casos clínicos, en ambos se confeccionó un colgajo en $L$ de base anterosuperior por fosa nasal derecha, escindiendo el piso de la fosa nasal y la región posterior del septum, $y$ un colgajo en $L$ de base anteroinferior por fosa nasal izquierda, al escindir la porción superior del septum por arriba del defecto y la región posterior del tabique por detrás de la perforación. Esta modificación a la técnica de Fairbanks permitió una adecuada rotación de los colgajos y así evitar las suturas a tensión.
En el segundo caso se utilizó una punta de tungsteno para realizar los colgajos. Esto mejoró considerablemente la hemostasia y visualización con endoscopio, así como un mejor trabajo en profundidad.

Se utilizó sutura de $\mathrm{PDS}^{\circledR}$ (polidioxanona) en ambos casos.

Esta sutura sintética reabsorbible de monofilamento trae la ventaja, por sobre otros elementos reabsorbibles, de una mayor fuerza tensil y mayor flexibilidad, además de degradarse por hidrolisis no enzimática. Es capaz de conservar el $70 \%$ de su resistencia tensil a los 14 días y el $25 \%$ a los 45 días. Tiene su absorción mínima a los 90 días, concluyendo a los 180 días $^{20}$.

Las suturas realizadas no deben ser a tensión, ya que así se logra evitar isquemia y necrosis.

Al finalizar las suturas se colocó Gelfoam ${ }^{\circledR}$, a la mucosa nasal reparada en ambos lados.

Las contraindicaciones para realizar la reparación de una perforación septal son la inhalación frecuente de cocaína, deportes de contacto y la presencia de comorbilidades (como granulomatosis de Wegener 0 vasculitis $)^{16}$.

En caso de presentarse recidivas, éstas son menores al defecto original, ocasionando menos molestias. Adicionalmente después de un tiempo prudente es posible repetir el procedimiento para intentar el cierre del defecto restante. Las razones de recidiva son: isquemia y necrosis de los colgajos, hematomas del tabique, infecciones posoperatorias 0 traumatismos ${ }^{16}$.

\section{CONCLUSIÓN}

Las perforaciones septales sintomáticas son un desafío para el otorrinolaringólogo. Existen numerosas técnicas quirúrgicas y la elección del tipo de cirugía depende del tamaño, de la topografía y de la experiencia del cirujano. La técnica de Fairbanks es aplicable por vía endonasal y presenta pocas complicaciones en manos expertas.

En nuestros dos casos, se realizaron modificaciones a la técnica de Fairbanks, con incisiones verticales, que permitieron una mejor rotación de colgajos. Por otro lado la visualización precisa con endoscopio permitió adecuada confección de colgajos y el trabajo en profundidad de la fosa nasal. 
Las limitaciones de este método vienen dadas por la altura del defecto y tamaño a reparar, no obstante es un método con altas tasas de éxito.

\section{BIBLIOGRAFÍA}

1. Castano R, Thériault G, Gautrin D. Categorizing nasal septal perforations of occupational origin as cases of corrosive rhinitis. Am J Ind Med 2007; 50(2): 150-3.

2. Re M, Paolucci L, Romeo R, Mallardi V. Surgical treatment of nasal septal perforations. Our experience. Acta Otorhinolaryngol Ital 2006; 26(2): 102-9.

3. Artal R, Urpegui A, Alfonso JI, Vallés H. Utilidad del botón septal y nivel de satisfacción obtenido en los pacientes con perforaciones del septum: Nuestra experiencia. Rev Otorrinolaringol Cir Cabeza Cuello 2011; 71: 145-54.

4. Dosen LK, HAYe R. Nasal septal perforation 19812005: Changes in etiology, gender and size. BMC Ear Nose Throath Disord 2007; 7: 1.

5. FaIRBAnks DN. Closure of nasal septal perforations. Arch Otolaryngol 1980; 106: 509-13.

6. Seyer BA, Grist W, Muller S. Aggressive destructive midfacial lesion from cocaine abuse. Oral Surg Oral Med Oral Pathol Oral Radiol Endod 2002; 44: 465-70.

7. Romo T, Sclafani AP, Falk AN, Toffel PH. A Graduated approach to the repair of nasal septal perforations. Plast Reconstr Surg 1999; 103: 66-75.

8. Andrés RF, Lohuis PJ, Vuyk HD. Nasal septum perforation repair using differently designed, bilateral intranasal flaps, with non opposing suture lines. J Plast Reconstr Aesthet Surg 2006; 59(8): 829-34.

9. Fairbanks DN, Fairbanks GR. Nasal septal perforation: prevention and management. Ann Plast Surg 1980; 6(5): 452-9.
10. Thomassin JM, Dessi P, Forman C, Danvin JB, Bailhache A. Cirugía de las perforaciones septales. EMC - Cirugía Otorrinolaringológica y Cervicofacial 2009; 10(1): 1-14.

11. Marshall AH, Johnston MN, Jones NS. Principles of septal correction. J Laryngol Otol 2004; 118(2): 129-34.

12. Blind A, Hulterström A, Berggren D. Treatment of nasal septal perforations with a custom-made Prosthesis. Eur Arch Otorhinolaryngol 2009; 266: 65-9.

13. KRIDEL RW. Septal perforation repair. Otolaryngol Clin North Am 1999; 32(4): 695-724.

14. Kridel RW, Appling WD, Wright WK. Septal perforation closure utilizing the external septorhinoplasty approach. Arch Otolaryngol Head Neck Surg 1986; 112(2): 168-72.

15. Schultz-Coulon HJ. Nasal septum repair-plasty with pedicle flap technique in 126 patients. An analysis. Laryngorhinootologie 1997; 76: 46674.

16. Neumann A, Morales-Minovi C, Schultz-Coulon $\mathrm{H}$. Cierre de las perforaciones del tabique nasal mediante colgajos «en puente». Acta Otorrinolaringol Esp 2011; 62(1): 31-9.

17. Pedroza F, Patrocinio LG, Arévalo O. A review of 25-year experience of nasal septal perforation repair. Arch Facial Plast Surg 2007; 9(1): 12-8.

18. Fairbanks DN, Chen SC. Closure of large nasal septum perforations. Arch Otolaryngol 1970; 91(5): 403-6.

19. Salama N, Oakley RJ, Skilbeck CJ, Choudhury N, $J_{A C O B} A$. Benefit from the minimally invasive sinus technique. J Laryngol Otol 2009; 123(2): 186-90.

20. Armas K, Armas B, Segura L, Márquez J, Armas K. Materiales de sutura quirúrgico. AMC [revista en Ia Internet]. 2009 Oct [citado 2013 Jul 29]; 13(5). Disponible en: http://scielo.sld. cu/scielo.php?script=sci_arttext\&pid=S102502552009000500011\&lng=es. 